\title{
The BRCAPRO 5.0 model is a useful tool in genetic counseling and clinical management of male breast cancer cases
}

\author{
Ines Zanna ${ }^{1}$, Piera Rizzolo ${ }^{2}$, Francesco Sera ${ }^{1}$, Mario Falchetti², Paolo Aretini ${ }^{1}$, Giuseppe Giannini ${ }^{2}$, \\ Giovanna Masala ${ }^{1}$, Alberto Gulino ${ }^{2}$, Domenico Palli ${ }^{\star, 1}$ and Laura Ottini ${ }^{2}$
}

No study has evaluated the performance of $B R C A 1 / 2$ mutations prediction models in male breast cancer (MBC) series. Although rare, $M B C$ deserves attention because male and female breast cancers share many characteristics, including the involvement of genetic predisposition factors such as BRCA1/BRCA2 mutations. Indeed, the occurrence of MBC is a commonly used criterion to select families for $B R C A$ mutation testing. We evaluated the performance and clinical effectiveness of four different predictive models in a population-based series of 102 Italian MBC patients characterized for BRCA1/2 mutations. Sensitivity, specificity, and positive and negative predictive values (PPV, NPV) were calculated for each risk model at the $10 \%$ threshold. The area under the ROC (AUC) curves and its corresponding asymptotic $95 \%$ Cls were calculated as a measure of the accuracy. In our study, the BRCAPRO version 5.0 had the highest combination of sensitivity, specificity, NPV and PPV for the combined probability and for the discrimination of BRCA2 mutations. In individuals with negative breast-ovarian cancer family history, BRCAPRO 5.0 reached a high discriminatory capacity $(A U C=0.92$ ) in predicting $B R C A 2$ mutations and showed values of sensitivity, specificity, NPV and PPV of $0.5,0.98,0.97$ and 0.67 , respectively, for the combined probability. BRCAPRO version 5.0 can be particularly useful in dealing with non-familial MBC, a circumstance that often represents a challenging situation in genetic counseling.

European Journal of Human Genetics (2010) 18, 856-858; doi:10.1038/ejhg.2010.29; published online 17 March 2010

Keywords: male breast cancer; BRCAPRO 5.0 prediction model; risk assessment

\section{INTRODUCTION}

Several models have been developed to assess the pre-test probability of identifying BRCA1/2 germline mutations in individuals at risk for hereditary breast and ovarian cancer. Widely used prediction models mainly include empirical risk assessment models, which predict the mutation probability based on variables describing personal or familial cancer history, such as Myriad II and Ontario Family History Assessment Tool (FHAT), ${ }^{1,2}$ and Mendelian models that predict the mutation probability based on penetrance and allelic frequencies of inherited alleles, such as BRCAPRO and the Italian Consortium (IC) model, a country-customized version of the BRCAPRO model. ${ }^{3-6}$ Recent studies carried out on various series of familial female breast cancers show that these models perform similarly. ${ }^{7-9}$ However, differences in performance can be observed depending on racial/ethnic groups. ${ }^{10,11}$ Thus, there is a need to develop an improved and broadly applicable version of risk assessment models. ${ }^{12,13}$

To the best of our knowledge, no study has evaluated the performance of $B R C A 1 / 2$ mutation prediction models in male breast cancer (MBC) series. MBC shares many similarities with female breast cancer, including the involvement of genetic predisposition factors such as BRCA1/2 mutations. ${ }^{14}$ In all, $4-40 \%$ of MBC cases are estimated to result from $B R C A 1$ and $B R C A 2$ mutations, BRCA2 mutations being more common. ${ }^{15}$ Approximately $20 \%$ of all MBC patients have a history of breast cancer (BC) in a first-degree female relative. ${ }^{15}$ Thus, the occurrence of $\mathrm{BC}$ in a male is a commonly used criterion to select families for $B R C A$ mutation testing. However, the majority of MBC patients do not have a positive family history (FH) of $\mathrm{BC}$ and our previous studies, performed on a large population-based series of MBCs, demonstrate that the majority of MBC cases are not accounted for by $B R C A 1 / 2$ alterations. ${ }^{16}$

\section{MATERIALS AND METHODS}

In this study, we evaluated the performance and clinical effectiveness of the above-described prediction models in a population-based series of 102 male patients affected by invasive $\mathrm{BC}$ and characterized for $B R C A 1 / 2$ mutations. The recruitment of the cases and the BRCA1/2 mutational analysis were carried out as recently described. ${ }^{16,17}$ Briefly, the recruitment of all incident MBC cases was based on all currently available local sources (including pathology departments and the hospital discharge database). For each case index the entire coding sequence and each intron-exon boundary were screened for germline mutations by combining the protein truncation test (PTT), single-strand conformation polymorphism (SSCP), multiplex ligation-dependent probe amplification (MLPA) and direct sequencing.

All MBC cases, diagnosed in the period 1991-2007, were resident in Eastern Tuscany (mean age at diagnosis $63.6 \pm 12.0$ years). For each study participant we obtained detailed information on his personal and familial history for cancer at any site. All information was collected by a geneticist and validated by

\footnotetext{
${ }^{1}$ Molecular and Nutritional Epidemiology Unit, Cancer Research and Prevention Institute-ISPO, Florence, Italy; ${ }^{2}$ Department of Experimental Medicine, Sapienza University of Rome, Rome, Italy

${ }^{*}$ Correspondence: Dr D Palli, Molecular and Nutritional Epidemiology Unit, Cancer Research and Prevention Institute-ISPO, Via Cosimo il Vecchio 2, Florence 50139, Italy. Tel: +39 05532697 828; Fax: +39 05532607 829; E-mail: d.palli@ispo.toscana.it
}

Received 7 September 2009; revised 14 January 2010; accepted 5 February 2010; published online 17 March 2010 
relevant sources, mainly local cancer and mortality registries. Overall, $38 \%$ (39/102) of the patients reported a first- and/or second-degree breast-ovarian cancer $\mathrm{FH}$, eight cases $(8 / 102,7.8 \%)$ carried a $B R C A 2$ mutation and two cases $(2 / 102,2.0 \%)$ carried the same founder BRCA1 mutation. ${ }^{16,18}$ Six of the 10 mutation carriers had a positive FH. Thus, mutation carriers account for $15.4 \%(6 / 39)$ of our male probands with a positive FH and for $6.4 \%(4 / 63)$ of those without a positive FH. For each proband we applied the Myriad II, FHAT, IC software version 3.4, and BRCAPRO models by using the CaGene version 4.2 and 5.0 software packages. The IC software utilizes country customized allele frequencies and penetrances obtained from Italian breast ovarian cancer families. ${ }^{5}$ In version 4.2 the genetic parameters have been updated as described by Chen et al. ${ }^{19}$ Version 5.0 of BRCAPRO has been recently updated by using new penetrance curves based on nine meta-analysis studies and by using male penetrance estimates based on the largest US cohort. ${ }^{20,21}$ Moreover, breast cancer biomarkers have been included.

All these models, except for Myriad and FHAT, give a composite probability as well as a BRCA1 or BRCA2 probability. We focused on evaluating the performance of the models that are frequently and easily used in clinical practice at our Institute and that can run through the CancerGene freeware counselling package. ${ }^{7-9}$

Sensitivity, specificity, PPV and NPV were calculated for each risk model at the $10 \%$ threshold. For the FHAT model this threshold corresponds to a cut-off score of 10 points. We rescaled the FHAT score as suggested by Parmigiani et al..$^{22}$ The area under the ROC (AUC) curves and its corresponding asymptotic 95\% CI were calculated as a measure of the accuracy of the model.

Table 1 Area under the ROC curve ( $C$-statistics) for each mode

\begin{tabular}{llcc}
\hline & \multicolumn{2}{c}{$95 \% \mathrm{Cl}$} \\
Test result variable & AUC & Lower bound & Upper bound \\
\hline Combined & & & \\
IC MODEL & 0.79 & 0.66 & 0.93 \\
BRCAPRO 4.2 & 0.78 & 0.64 & 0.92 \\
BRCAPRO 5.0 & 0.82 & 0.67 & 0.97 \\
FHAT & 0.72 & 0.53 & 0.91 \\
MYRIAD & 0.61 & 0.40 & 0.82 \\
BRCA2 & & & \\
IC MODEL & 0.80 & 0.65 & 0.94 \\
BRCAPRO 4.2 & 0.79 & 0.64 & 0.94 \\
BRCAPRO 5.0 & 0.83 & 0.71 & 0.95 \\
\hline
\end{tabular}

Abbreviation: ROC, receiver operator characteristic.

\section{RESULTS}

As shown by AUC estimates (Table 1), all Mendelian models, but not empirical models, performed equally well, and their ability to discriminate between mutation-carrying and non-carrying probands was similar. On comparing the sensitivity, specificity, PPV and NPV, we observed that NPV was very high for all models (range 0.97-1.0). On the contrary, considering the low prevalence of $B R C A$ mutation in the general population, it is not surprising that the PPV at $10 \%$ threshold was low (range 0.11-0.29), as demonstrated in studies performed on females. ${ }^{23}$ Interestingly, in our study, the BRCAPRO version 5.0 had the highest combination of sensitivity, specificity, NPV and PPV not only for the combined probability but also for the discrimination of BRCA2 mutations (Table 2).

We also conducted an analysis stratified for breast-ovarian cancer FH. No difference in performance of the models was observed in MBC cases with positive FH (range of AUC for Mendelian models: 0.78-0.84). Actually, none of the models performed better than the others and their ability to discriminate between mutation-carrying and non-carrying probands in the subset of MBC patients with positive $\mathrm{FH}$ was similar. It was instead observed that, in men with a negative $\mathrm{FH}, \mathrm{BRCAPRO}$ version 5.0 reached a high discriminatory capacity $(A U C=0.92)$ in predicting $B R C A 2$ mutations and showed values of sensitivity, specificity, NPV and PPV of 0.50, 0.98, 0.97 and 0.67, respectively, for the combined probability. BRCAPRO 5.0 may then be deemed to be particularly useful in dealing with MBC patients with a negative $\mathrm{FH}$, a circumstance that may represent a challenge in genetic counseling as non-familial MBCs are the vast majority.

\section{DISCUSSION}

An effective model must be accurate and hands-on. On comparing positive and negative likelihood ratios (BRCAPRO 4.2 1.77, 0; BRCAPRO 5.0 3.87, 0.25; IC model 1.20, 0; FHAT 2.6, 0.41), it was evident that none of the models could rule or rule out the $B R C A$ mutation carrier status of a MBC case. Nevertheless, although the BRCAPRO model requires computer access and is not easily accessible during genetic counseling, being rather cumbersome for data entry, version 5.0 of this model represents a helpful tool in management of $\mathrm{MBC}$ cases with a negative FH. Indeed, when a cut-off value $\geqslant 10 \%$ was considered, BRCAPRO 5.0 fully succeeded in identifying mutation carriers with no $\mathrm{FH}$ in our MBC series. In particular, 3 of the 63 MBC cases with a negative $\mathrm{FH}(3 / 63,4.8 \%)$ showed a BRCAPRO 5.0 value $\geqslant 10 \%$ and 2 out of these $3(67 \%)$ were mutation carriers. This

Table 2 Performance measures for each model at the $10 \%$ threshold

\begin{tabular}{|c|c|c|c|c|c|c|}
\hline & \multicolumn{2}{|c|}{ Proportion of carriers by model probability } & \multicolumn{4}{|c|}{ Test parameters at $10 \%$ threshold $(95 \% \mathrm{Cl})$} \\
\hline & $<10 \%$ & $\geqslant 10 \%$ & Sensitivity & Specificity & $P P V$ & $N P V$ \\
\hline \multicolumn{7}{|l|}{ Combined } \\
\hline IC MODEL & $0 / 13$ & $10 / 89$ & 1.0 & 0.16 & 0.11 & 1.0 \\
\hline BRCAPRO 4.2 & $0 / 37$ & $10 / 65$ & 1.0 & 0.44 & 0.15 & 1.0 \\
\hline BRCAPRO 5.0 & $2 / 73$ & $8 / 28$ & 0.80 & 0.78 & 0.29 & 0.97 \\
\hline FHAT & $3 / 77$ & $7 / 25$ & 0.70 & 0.73 & 0.28 & 0.96 \\
\hline MYRIAD & 0/0 & $10 / 102$ & 1.0 & 0.0 & 0.10 & - \\
\hline \multicolumn{7}{|l|}{ BRCA2 } \\
\hline IC MODEL & $0 / 15$ & $8 / 87$ & 1.0 & 0.18 & 0.09 & 1.0 \\
\hline BRCAPRO 4.2 & $0 / 41$ & $8 / 61$ & 1.0 & 0.46 & 0.13 & 1.0 \\
\hline BRCAPRO 5.0 & $3 / 76$ & $5 / 26$ & 0.63 & 0.78 & 0.19 & 0.96 \\
\hline
\end{tabular}

Abbreviations: NPV, negative predictive value; PPV, positive predictive value. 
means that, in clinical practice, in $\mathrm{MBC}$ patients with a negative $\mathrm{FH}$, genetic testing can be reasonably restricted to affected subjects with a BRCAPRO 5.0 value $\geqslant 10 \%$, thus allowing for saving limited healthcare resources. By contrast, of the $60 \mathrm{MBC}$ cases with a negative $\mathrm{FH}$ and with a BRCAPRO 5.0 value $<10 \%$, only $2(3 \%)$ were mutation carriers. However, when examining in detail these two carriers, the presence of a positive FH could not be fully excluded in both cases. In fact, for one case (BRCAPRO:0.082), who reported a FH positive for colon cancer, it was not possible to obtain complete information about his family (both sides), whereas a strongly positive third-degree $\mathrm{FH}$, not evaluated by the CaGene implementation of BRCAPRO, emerged for the second case (BRCAPRO:0.034). Intriguingly, this latter case was affected by a triple-negative tumor (ER-, PR-, and HER2-) and was a BRCA1 carrier. Thus, following the clinical judgment, both $\mathrm{MBC}$ cases should have been eligible for BRCA testing. Overall, these observations point to the importance of acquiring extensive and accurate data on $\mathrm{FH}$ and of using predictive models that can handle third-degree relationships, such as the $\mathrm{R}$ version of BRCAPRO, ${ }^{19}$ in evaluating BRCA mutation probability in $\mathrm{MBC}$. Furthermore, our results point to the relevance of tumour pathological features to be considered in evaluating BRCA1/2 mutation probability. Indeed, BRCAPRO 5.0 takes into account immunohistochemical data regarding ER, PR, CK14 and CK5.6, but not HER2, expression. In this context, it is noteworthy that we have recently described the phenotypic characteristics of $B R C A 1 / 2$-related MBCs and shown that a specific phenotype that included HER2 over-expression was associated with BRCA2-related MBCs. ${ }^{16}$

In conclusion, the BRCAPRO 5.0 model, together with an experienced clinical evaluation, is a useful tool in selecting cases of male breast cancer for mutation analysis in the presence of $\mathrm{MBC}$ cases with a negative FH. In this context, the BRCAPRO 5.0 model performs better in taking account of personal history of cancer and selected tumour characteristics. On the other hand, a smaller family size probably has a role in modulating the mutation probability assigned by the model.

Far from being limited to the rare $\mathrm{MBC}$ disease, the possibility of improving the identification of $B R C A$ mutation carriers in $\mathrm{MBC}$ also impacts on female relatives of cases who are often addressed to Genetic Counseling Services to be informed about their BC risk and more adequate clinical surveillance.

\section{CONFLICT OF INTEREST}

The authors declare no conflict of interest.

\section{ACKNOWLEDGEMENTS}

This study was supported by Regione Toscana in the frame of the High-Risk Cancer Family Project and by a grant from Associazione Italiana per la Ricerca sul Cancro (AIRC).
1 Frank TS, Deffenbaugh AM, Reid JE et al: Clinical characteristics of individuals with germline mutations in BRCA1 and BRCA2: analysis of 10000 individuals. J Clin Oncol 2002; 20: 1480-1490.

2 Gilpin CA, Carson N, Hunter AG: A preliminary validation of a family history assessment form to select women at risk for breast or ovarian cancer for referral to a genetics center. Clin Genet 2000; 58: 299-308.

3 Parmigiani G, Berry D, Aguilar O: Determining carrier probabilities for breast cancersusceptibility genes BRCA1 and BRCA2. Am J Hum Genet 1998; 62: 145-158.

4 Berry DA, Iversen Jr ES, Gudbjartsson DF et al: BRCAPRO validation, sensitivity of genetic testing of BRCA1/BRCA2, and prevalence of other breast cancer susceptibility genes. J Clin Oncol 2002; 20: 2701-2712.

5 Marroni F, Aretini P, D'Andrea E et al: Penetrances of breast and ovarian cancer in a large series of families tested for BRCA1/2 mutations. Eur J Hum Genet 2004; 12 : 899-906.

6 Capalbo C, Ricevuto E, Vestri A et al: Improving the accuracy of BRCA1/2 mutation prediction: validation of the novel country-customized IC software. Eur J Hum Genet 2006; 14: 49-54.

7 Barcenas $\mathrm{CH}$, Hosain GM, Arun B et al: Assessing BRCA carrier probabilities in extended families. J Clin Oncol 2006; 24: 354-360.

8 Antoniou AC, Durocher F, Smith P, Simard J, Easton DF: BRCA1 and BRCA2 mutation predictions using the BOADICEA and BRCAPRO models and penetrance estimation in high-risk French-Canadian families. Breast Cancer Res 2006; 8: R3.

9 Panchal SM, Ennis M, Canon S, Bordeleau LJ: Selecting a BRCA risk assessment model for use in a familial cancer clinic. BMC Med Genet 2008; 9: 116.

10 Kurian AW, Gong GD, Chun NM et al: Performance of BRCA1/2 mutation prediction models in Asian Americans. J Clin Oncol 2008; 26: 4752-4758.

11 Kurian AW, Gong GD, John EM et al: Performance of prediction models for BRCA mutation carriage in three racial/ethnic groups: findings from the Northern California Breast Cancer Family Registry. Cancer Epidemiol Biomarkers Prev 2009; 18: 1084-1091.

12 Chen S, Blackford AL, Parmigiani G: Tailoring BRCAPRO to Asian-Americans. J Clin Oncol 2009; 27: 642-643; author reply 643-644.

13 Pasanisi P, Hedelin G, Berrino J et al: Oral contraceptive use and BRCA penetrance: a case-only study. Cancer Epidemiol Biomarkers Prev 2009; 18: 2107-2113.

14 Brinton LA, Richesson DA, Gierach GL et al: Prospective evaluation of risk factors for male breast cancer. J Natl Cancer Inst 2008; 100: 1477-1481.

15 Ottini L, Palli D, Rizzo S, Federico M, Bazan V, Russo A: Male breast cancer. Crit Rev Oncol Hematol 2010; 73: 141-155.

16 Ottini L, Rizzolo P, Zanna I et al: BRCA1/BRCA2 mutation status and clinicalpathologic features of 108 male breast cancer cases from Tuscany: a populationbased study in central Italy. Breast Cancer Res Treat 2009; 116: 577-586.

17 Falchetti M, Lupi R, Rizzolo $\mathrm{P}$ et al: BRCA1/BRCA2 rearrangements and CHEK2 common mutations are infrequent in Italian male breast cancer cases. Breast Cancer Res Treat 2008; 110: 161-167.

18 Papi L, Putignano AL, Congregati $C$ et al: Founder mutations account for the majority of BRCA1-attributable hereditary breast/ovarian cancer cases in a population from Tuscany, Central Italy. Breast Cancer Res Treat 2009; 117: 497-504.

19 Chen S, Wang W, Broman KW et al: BayesMendel: an R Environment for Mendelian risk prediction. Stat Appl Genet $\mathrm{Mol}$ Biol 2004: 3: 1-16.

20 Chen S, Parmigiani G: Meta-analysis of BRCA1 and BRCA2 penetrance. J Clin Oncol 2007; 25: 1329-1333.

21 Tai YC, Domchek S, Parmigiani G, Chen S: Breast cancer risk among male BRCA1 and BRCA2 mutation carriers. J Nat/ Cancer Inst 2007; 99: 1811-1814.

22 Parmigiani G, Chen S, Iversen Jr ES et al: Validity of models for predicting BRCA 1 and BRCA2 mutations. Ann Intern Med 2007; 147: 441-450.

23 Kang HH, Williams R, Leary J, Ringland C, Kirk J, Ward R: Evaluation of models to predict BRCA germline mutations. Br J Cancer 2006; 95: 914-920. 